\title{
Extremely Efficient and Robust
}

\author{
A new high-frequency generator combines an efficiency rate of $80 \%$ with a high level \\ of reliability to give significant cost savings.
}

$E_{f}^{\mathrm{f}}$ fficiency and a robust design are two -features of high-frequency power supplies which in the past could not be combined. Users had the choice between stable, less efficient generators with class A or B amplifiers or efficient but sensitive machines with class $\mathrm{E}$ switch-mode power supplies.

With the arrival of the new TruPlasma RF 3012 high-frequency generator from Trumpf Hüttinger, users no longer need to compromise. It offers an efficiency level of $80 \%$ and is also highly robust and reliable. All of this is made possible by class $\mathrm{D}$ converter topology, together with a high-frequency combiner.

The generator has a power output of 12 kilowatts with a frequency of 13.56 megahertz and is ideal for PECVD, etching and RF sputter processes in the flat panel display, solar power and semiconductor industries. These processes become significantly more efficient with the new generator, which leads to a considerable reduction in costs.

\section{Innovative converter topology}

The power loss of the new generator with its class D converter topology is

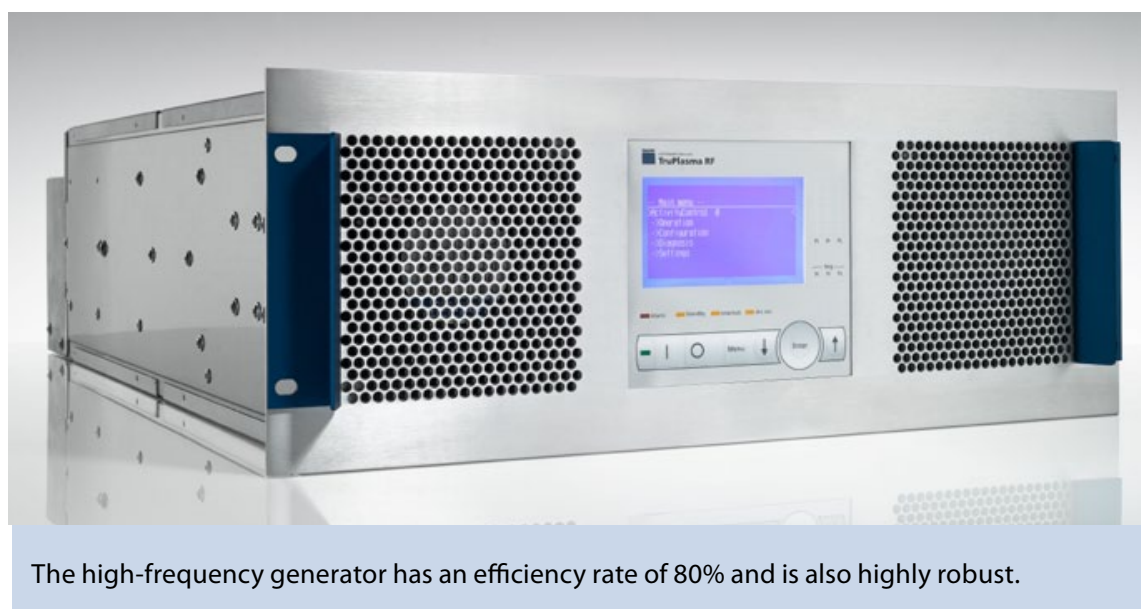

around half that of the power supplies currently on the market, which have an efficiency rate of $60 \%$.

In a typical PECVD coating machine, which applies passive SiNx coatings to wafer-based solar cells, the generator often operates for up to 22 hours a day on 355 days of the year. At current electricity prices, the increased efficiency results in a cost saving of around 3500 euros a year per generator.

The $50 \%$ reduction in power loss also means that less energy is needed to cool the generator. This in turn leads to a reduction in water consumption and in the investment required in the in-

\section{Robust and reliable}

The CombineLine high-frequency combiner system is what makes the generator so robust. CombineLine combines the power from the internal transistor modules and feeds it into the output. Reflected power as a result of mismatches is passed to a water-cooled resistor. This ensures that the transistor modules are fully protected and allows the generator to absorb the reflected power without problems. The result is frastructure. an extremely robust machine that also provides high levels of availability. In addition, CombineLine has true 50ohm output impedance and can balance out fluctuations at any point in the process.

This is where it differs significantly from conventional RF switch-mode power supplies, which under certain conditions amplify small process fluctuations and prevent stable operation. In order to avoid unstable processes of this kind, users must adjust the length of the cable between the generator and the vacuum chamber to suit the process. This is laborious and time-consuming and can cause problems when the process conditions change.

With CombineLine, this work is no longer needed. The generator can be operated using a cable of any length, which increases its flexibility and enables it to be integrated into existing systems, saving both space and money.

A further benefit of the new generator is its wide range of communication ports. Alongside the usual analogue, RS 232, Devicenet and Profibus interfaces, it also has an EtherCAT port. EtherCAT will soon be the new market standard. As a result, the generator is already able to accommodate the bus systems of the future. This means that users can prepare their systems today for the market standard of tomorrow.

The new generator also meets growing demands for reliable RF arc management. With its fast, technically advanced arc management system, it can accommodate even highly challenging processes and is fully equipped for the applications of the future.

\section{Contact:}

Trump Hüttinger GmbH + Co. KG, Freiburg, Germany, Tel. +497618971 0, www.trumpf-huettinger.com 